\title{
Early diagnosis and antibiotic treatment for fulminant Clostridium difficile infection
}

\author{
Kazutaka Fukushima ${ }^{1}$ \\ Kent Doi ${ }^{1}$ \\ Ryota Inokuchi ${ }^{2}$ \\ Ichiro Hirayama ${ }^{1}$ \\ | Takahiro Hiruma ${ }^{1}$ |
}

${ }^{1}$ Department of Emergency and Critical Care Medicine, The University of Tokyo Hospital, Bunkyo-ku, Japan

${ }^{2}$ Department of Health Services Research, Faculty of Medicine, University of

Tsukuba, Tsukuba, Japan

\section{Correspondence}

Ryota Inokuchi, Department of Health Services Research, Faculty of Medicine, University of Tsukuba, 1-1-1 Tenno-dai, Tsukuba, Ibaraki 305-8575, Japan. Email: intensivecareunits@gmail.com

\begin{abstract}
In fulminant Clostridium difficile infection (CDI), early diagnosis is important, and early diagnosis could save fulminant CDI patients that do not qualify for surgery due to severe complicating conditions by conservative antibiotic therapy.
\end{abstract}

\section{K E Y W O R D S}

colitis, enzyme immunoassay, toxin

\section{1 | INTRODUCTION}

The guidelines recommend surgical treatment for fulminant Clostridium difficile infection (CDI). However, mortality remains high, and patients with severe condition are not considered for surgical treatment because of poor post-surgical outcomes. Moreover, delayed diagnosis of fulminant CDI may induce intestinal necrosis. Thus, early diagnosis is crucial.

Fulminant CDI has a low incidence rate of about $3 \%$ of the total CDI cases. ${ }^{1}$ However, fulminant CDI is associated with high morbidity and mortality. The optimal time for surgery is within 48 hours after initiating conservative treatment without seeing a response and developing multiple organ failure or bowel perforation. ${ }^{2}$ When toxic megacolon or perforation is present, subtotal colectomy with ileostomy remains the standard of care.

However, this treatment can lead to mortality rates approaching $\sim 50 \%{ }^{3}$ Furthermore, patients with acute respiratory failure with intubation, shock requiring vasopressors, age greater than 80 years, and dialysis-dependent renal failure are not considered surgical candidates as they have conditions that predict poor post-surgical outcomes. ${ }^{4}$ In this manuscript, we described that early diagnosis and conservative antibiotic treatment saved a dialysis-dependent patient with fulminant CDI, intubation, and septic shock.

\section{2 | CASE REPORT}

A 74-year-old Japanese man with type 2 diabetes mellitus was transferred to our hospital from another hospital because of persistent watery diarrhea, disturbed consciousness, and shock. Fifteen days before the visit, he underwent pyloric gastrectomy for advanced gastric cancer. After the operation, he suffered from pneumonia and was treated with antibiotics. Two days before the visit, he experienced large amounts of watery stool, followed by fever and shock. However, the enzyme immunoassays for toxin $\mathrm{A} / \mathrm{B}$ were negative.

Upon admission to our hospital, his vital signs were as follows: blood pressure $(88 / 54 \mathrm{~mm} \mathrm{Hg})$, pulse (115 beats per minute), body temperature $\left(39.2^{\circ} \mathrm{C}\right)$, respiratory rate 
(40 breaths per minute), Glasgow coma scale eye opening (2), verbal response (2), and motor response (1). Blood tests demonstrated high inflammatory response, acute kidney injury, hypokalemia, and elevated serum creatine kinase. Abdominal computed tomography (CT) scans showed accordion sign (Figure 1).

After the patient was admitted to the intensive care unit, tracheal intubation, administration of meropenem for septic shock of unknown cause and noradrenaline $(0.6 \mu \mathrm{g} / \mathrm{kg} / \mathrm{min}$ ute) to maintain blood pressure, continuous renal replacement therapy for anuria, and severe metabolic acidosis were performed. In addition, we submitted the patient's stool sample for enzyme immunoassay (EIA) for toxin A/B and glutamate dehydrogenase (GDH) test. On day 2, his white blood cell count was elevated to $76,400 / \mu \mathrm{L}$ and his lactic acid level reached $5.7 \mathrm{mmol} / \mathrm{L}$. Moreover, the results for enzyme immunoassay for toxin A/B and the GDH test were positive. Thus, we diagnosed fulminant Clostridium difficile infection.

Surgical treatment was not considered because the patient has the following conditions: intubation, shock requiring vasopressors, and dialysis-dependent renal failure. The antibiotics were changed from meropenem to metronidazole and added to an oral administration of vancomycin.

Thereafter, the patient's general condition gradually improved. On day 5, watery diarrhea disappeared and intestinal peristalsis improved. In addition, administration of noradrenaline and continuous renal replacement therapy were removed, and he was successfully extubated. After discontinuing the administration of antimicrobial agents, we confirmed that the patient did not have CDI relapse. On day 49 , he returned to his former hospital without any complications.

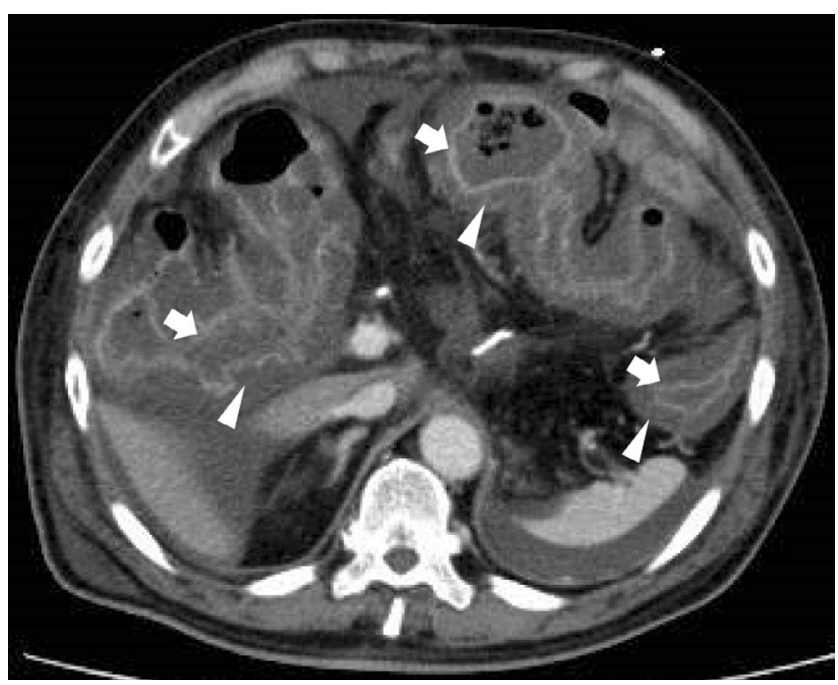

FIG URE 1 Abdominal computed tomography image showed accordion sign; high-attenuation oral contrast in the colonic lumen (arrows) alternating with low-attenuation inflamed mucosa (arrowheads)

\section{DISCUSSION}

We showed that the patient with fulminant CDI was saved by early diagnosis and conservative antibiotic treatment. Late diagnosis could cause megacolon and intestinal perforation. ${ }^{5}$ Thus, early diagnosis is crucial before the disease progresses. However, early diagnosis for CDI is difficult, because there is no single stool test, and the best standard laboratory test for CDI diagnosis has not been clearly established. ${ }^{6}$ In general hospitals, the EIA for toxin $\mathrm{A} / \mathrm{B}$ test has been adopted because it is fast, convenient, and inexpensive. ${ }^{7}$ However, the EIA for toxin A/B has a sensitivity of 32-98\% and a specificity of $84-100 \%$, and thus, we repeated toxin A/B EIA combined with GDH test. ${ }^{8}$ GDH screening for C. difficile used in association with EIA for toxin A/B quickly gives an accurate test result. ${ }^{7,8}$ In addition, abdominal $\mathrm{CT}$ has been studied as an imaging modality for diagnosing CDI. ${ }^{9}$ Typical CT findings of colonic wall thickening (most common), dilation, peri-colonic stranding, accordion sign, double-halo sign, and target sign are nonspecific and can be found in other forms of colitis. However, these may be more pronounced with CDI. ${ }^{10}$

\section{CONCLUSION}

In cases with high clinical suspicion for fulminant CDI, physicians should combine the examinations for early diagnosis and conservative therapy to reduce mortality in patients with fulminant CDI.

\section{CONFLICT OF INTEREST}

None declared.

\section{AUTHOR CONTRIBUTIONS}

$\mathrm{KF}, \mathrm{IH}, \mathrm{TH}$, and $\mathrm{KD}$ : contributed to management of the patient. KF: drafted an initial manuscript. IH, RI, and KD: critically reviewed the manuscript. All the authors contributed to writing the manuscript and have provided written consent for publication.

\section{ETHICAL APPROVAL}

Need for ethical approval waived. Consent from the patient deemed to be enough.

\section{CONSENT FOR PUBLICATION}

Written informed consent was obtained from the patient for publication of this case report and any accompanying images. A copy of the written consent is available for review by the editor-in-chief of this journal.

\section{DATA AVAILABILITY STATEMENT}

Data sharing was not applicable to this article as no datasets were generated or analyzed during the current study. 


\section{ORCID}

Ryota Inokuchi (D) https://orcid.org/0000-0001-6343-2298

\section{REFERENCES}

1. Napolitano LM, Edmiston CE Jr. Clostridium difficile disease: diagnosis, pathogenesis, and treatment update. Surgery. 2017;162:325-348.

2. Dallal M, Harbrecht G, Bojoukas J, et al. Fulminant Clostridium difficile: an underappreciated and increasing cause of death and complications. Ann Surg. 2001;235:363-372.

3. Fischer M, Sipe B, Cheng YW, et al. Fecal microbiota transplant in severe and severe-complicated Clostridium difficile: a promising treatment approach. Gut Microbes. 2017;8:289-302.

4. Hall JF, Berger D. Outcome of colectomy for Clostridium difficile colitis: a plea for early surgical management. Am J Surg. 2008;196:384-388.

5. Greenstein AJ, Byrn JC, Zhang LP, Swedish KA, Jahn AE, Divino CM. Risk factors for the development of fulminant Clostridium difficile colitis. Surgery. 2008;143:623-629.

6. Carroll KC. Tests for the diagnosis of Clostridium difficile infection: the next generation. Anaerobe. 2011;17:170-174.
7. Planche T, Aghaizu A, Holliman R, et al. Diagnosis of Clostridium difficile infection by toxin detection kits: a systematic review. Lancet Infect Dis. 2008;8:777-784.

8. Brecher SM, Novak-Weekley SM, Nagy E. Laboratory diagnosis of Clostridium difficile infections: there is light at the end of the colon. Clin Infect Dis. 2013;57:1175-1181.

9. Ros PR, Buetow PC, Pantograg-Brown L, Forsmark CE, Sobin LH. Pseudomembranous colitis. Radiology. 1996;198:1-9.

10. Paláu-Dávila L, Lara-Medrano R, Negreros-Osuna AA, et al. Efficacy of computed tomography for the prediction of colectomy and mortality in patients with clostridium difficile infection. Ann Med Surg (Lond). 2016;12:101-105.

How to cite this article: Fukushima K, Inokuchi R, Hirayama I, Hiruma T, Doi K. Early diagnosis and antibiotic treatment for fulminant Clostridium difficile infection. Clin Case Rep. 2021;9:729-731. https://doi. org/10.1002/ccr3.3613 